\title{
Transverse spherocity dependence of azimuthal anisotropy in heavy-ion collisions at the LHC using a multi-phase transport model
}

\author{
Neelkamal Mallick, ${ }^{a, *}$ Raghunth Sahoo, ${ }^{a, b}$ Sushanta Tripathy ${ }^{c}$ and Antonio Ortiz ${ }^{d}$ \\ ${ }^{a}$ Department of Physics, Indian Institute of Technology Indore, Simrol, Indore 453552, India \\ ${ }^{b}$ CERN, CH 1211, Geneva 23, Switzerland \\ ${ }^{c}$ INFN - sezione di Bologna, via Irnerio 46, 40126 Bologna BO, Italy \\ ${ }^{d}$ Instituto de Ciencias Nucleares, Universidad Nacional Autónoma de México, México Distrito Federal \\ 04510, México \\ E-mail: Neelkamal.Mallick@cern.ch, Raghunath.Sahoo@cern.ch, \\ Sushanta.Tripathy@cern.ch, Antonio.Ortiz.Velasquez@cern.ch
}

One of the event shape observables, the transverse spherocity $\left(S_{0}\right)$, has been studied successfully in small collision systems such as proton-proton collisions at the LHC as a tool to separate jetty and isotropic events. It has a unique capability to distinguish events based on their geometrical shapes. In our work, we report the first implementation of transverse spherocity in heavyion collisions using a multi-phase transport model (AMPT). We have performed an extensive study of azimuthal anisotropy of charged particles produced in heavy-ion collisions as a function of transverse spherocity $\left(S_{0}\right)$. We have followed the two-particle correlation (2PC) method to estimate the elliptic flow $\left(v_{2}\right)$ in different centrality classes in $\mathrm{Pb}-\mathrm{Pb}$ collisions at $\sqrt{s_{\mathrm{NN}}}=5.02 \mathrm{TeV}$ for high- $S_{0}, S_{0}$-integrated and low- $S_{0}$ events. We found that transverse spherocity successfully differentiates heavy-ion collisions' event topology based on their geometrical shapes, i.e., high and low values of spherocity. The high- $S_{0}$ events have nearly zero elliptic flow, while the low- $S_{0}$ events contribute significantly to the elliptic flow of spherocity-integrated events.

The Ninth Annual Conference on Large Hadron Collider Physics - LHCP2021

7-12 June 2021

Online

\footnotetext{
${ }^{*}$ Speaker
} 


\section{Introduction}

The production of a hot and dense, deconfined state of matter known as the quark-gluon plasma (QGP) has already been established in heavy-ion collisions at the Large Hadron Collider (LHC) at CERN, Switzerland, and Relativistic Heavy Ion Collider (RHIC) at BNL, USA. Recent studies at the LHC show heavy-ion-like features such as ridge-like structures [1] and strangeness enhancements [2] in $p p$ collisions. To understand the system dynamics and production of jets in $p p$ collisions, an event shape observable, known as the transverse spherocity $\left(S_{0}\right)$, has been introduced recently at the LHC [3-7]. These studies show that transverse spherocity $\left(S_{0}\right)$ has unique capabilities to distinguish events based on their geometrical shapes i.e. jetty and isotropic events. The study of transverse spherocity in heavy-ion collisions may reveal new and unique physics results where the formation of QGP is already known. This study in heavy-ion collisions shall also complement the current event shape approach based on flow vector analysis at the LHC [8, 9].

In this work [10], we report the first implementation of transverse spherocity in heavy-ion collisions using a multi-phase transport (AMPT) model [11]. We have performed an extensive study of azimuthal anisotropy of charged particles produced in $\mathrm{Pb}-\mathrm{Pb}$ collisions at $\sqrt{s_{\mathrm{NN}}}=5.02 \mathrm{TeV}$ as a function of transverse spherocity $\left(S_{0}\right)$. We have followed the two-particle correlation (2PC) method to estimate the elliptic flow $\left(v_{2}\right)$ and subtract non-flow from our calculations by following standard experimental procedures.
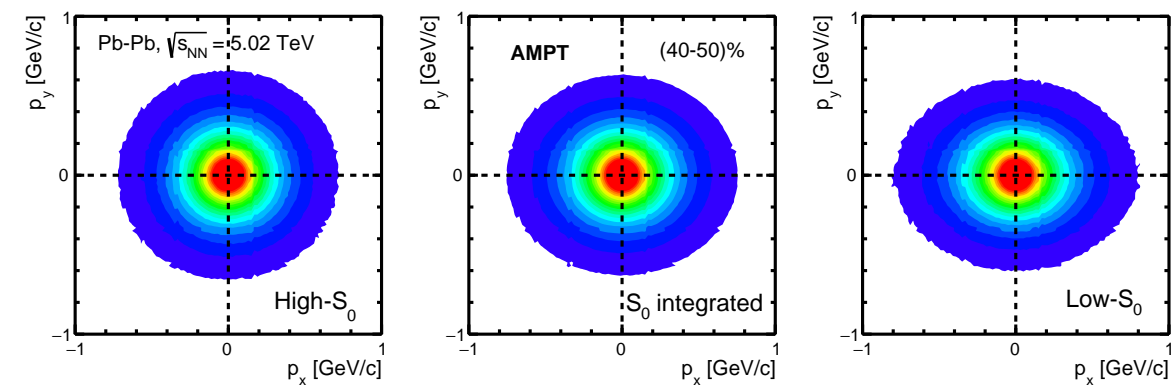

Figure 1: (Color Online) Transverse momentum space correlation $\left(p_{\mathrm{y}}\right.$ vs. $\left.p_{\mathrm{x}}\right)$ for different spherocity classes in (40-50)\% central $\mathrm{Pb}-\mathrm{Pb}$ collisions at $\sqrt{s_{\mathrm{NN}}}=5.02 \mathrm{TeV}$ using AMPT model. (Fig. 2 [10])

Transverse spherocity $\left(S_{0}\right)$ is an event property which is also a collinear and infrared safe quantity [3-5] and defined as follows,

$$
S_{0}=\frac{\pi^{2}}{4}\left(\frac{\Sigma_{i}\left|\vec{p}_{T_{i}} \times \hat{n}\right|}{\Sigma_{i} p_{T_{i}}}\right)^{2}
$$

Here, $\hat{n}\left(n_{T}, 0\right)$ is a unit vector known as the spherocity axis, which minimizes the ratio in Eq. 1 . For jetty events $S_{0} \approx 0$ and for isotropic events $S_{0} \approx 1$ [7]. We have used the mid-rapidity $(|\eta|<0.8)$ spherocity distribution with $p_{\mathrm{T}}>0.15 \mathrm{GeV} / \mathrm{c}$ with events having at least five such tracks to meet similar conditions as in the ALICE experiment at the LHC. The low- $S_{0}$ and high- $S_{0}$ events represent the events lying in the bottom $20 \%$ and top $20 \%$ in the $S_{0}$ distributions whereas $S_{0}$-integrated events take all events into account. 
Figure 1 represents the transverse momentum space correlation ( $p_{\mathrm{y}}$ vs. $p_{\mathrm{x}}$ ) for high- $S_{0}, S_{0^{-}}$ integrated and low- $S_{0}$ events in (40-50)\% central Pb-Pb collisions at $\sqrt{s_{\mathrm{NN}}}=5.02 \mathrm{TeV}$ using AMPT model. The elliptic flow in $S_{0}$-integrated events can be clearly seen from the elliptic shape of the correlation plot. This is indeed credited to the initial pressure gradient caused due to the initial almond-shaped nuclear overlap region in semi-central collisions, which is then translated to the momentum space $\left(p_{\mathrm{x}}>p_{\mathrm{y}}\right)$ correlations. The interesting thing is that the high- $S_{0}$ events show almost spherical momentum correlation indicating the presence of nearly zero elliptic flow in such events. In contrast, the events with low- $S_{0}$ show a greater elliptical shape correlation. That indicates low- $S_{0}$ events should be more elliptic and therefore contribute more towards $v_{2}$.

\section{Results and Discussions}

To estimate the elliptic flow we have used the two-particle correlation method (2PC) $[8,12]$. The two particle correlation function $C(\Delta \eta, \Delta \phi)$ is constructed by taking the ratios of same-event pairs $S(\Delta \eta, \Delta \phi)$ to mixed-event pairs $B(\Delta \eta, \Delta \phi)$ given by,

$$
C(\Delta \eta, \Delta \phi)=\frac{S(\Delta \eta, \Delta \phi)}{B(\Delta \eta, \Delta \phi)}
$$
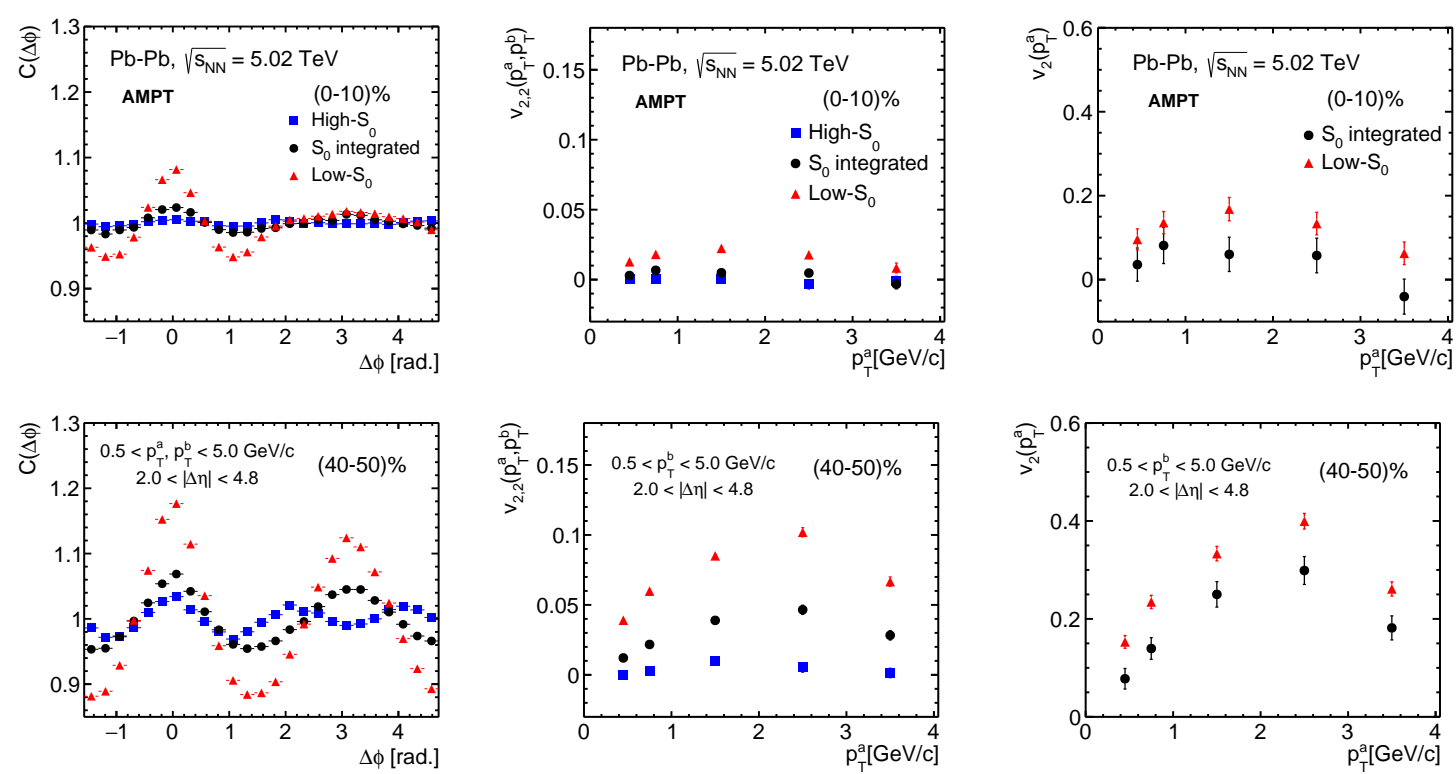

Figure 2: (Color Online) First column: One dimensional azimuthal correlation of charged particles, second column: two particle elliptic flow coefficient $\left(v_{2,2}\left(p_{T}^{a}, p_{T}^{b}\right)\right)$ of charged particles as a function of $p_{T}^{a}$, and third column: single particle elliptic flow coefficient $\left(v_{2}\left(p_{T}^{a}\right)\right)$ of charged particles as a function of $p_{T}^{a}$ for low- $S_{0}$, high- $S_{0}$ and $S_{0}$-integrated events in $\mathrm{Pb}-\mathrm{Pb}$ collisions at $\sqrt{s_{\mathrm{NN}}}=5.02 \mathrm{TeV}$ for $0 \%-10 \%$ (top), 40\%-50\% (bottom) centrality classes using AMPT model. (Figs. 4-6 [10])

The one dimensional correlation function $(C(\Delta \phi))$ is then calculated by integrating all the pairs with pseudorapidity gap $2.0<|\Delta \eta|<4$.8. This step helps in reducing the contributions from 
non-flow effects [8]. From the $C(\Delta \phi)$ distribution, the two particle flow coefficient $\left(v_{2,2}\left(p_{\mathrm{T}}^{a}, p_{\mathrm{T}}^{b}\right)\right)$ could be easily obtained as [13-15],

$$
v_{n, n}\left(p_{\mathrm{T}}^{a}, p_{\mathrm{T}}^{b}\right)=\langle\cos (n \Delta \phi)\rangle=\frac{\sum_{m=1}^{N} \cos \left(n \Delta \phi_{m}\right) \times C\left(\Delta \phi_{m}\right)}{\sum_{m=1}^{N} C\left(\Delta \phi_{m}\right)} .
$$

where, $N=200$ is the number of $\Delta \phi$ bins in the range $-\pi / 2<\Delta \phi<3 \pi / 2$. Here, $v_{n, n}$ are symmetric functions with respect to $p_{\mathrm{T}}^{a}$ and $p_{\mathrm{T}}^{b}$. The single particle flow coefficient could be obtained as,

$$
v_{n, n}\left(p_{\mathrm{T}}^{a}, p_{\mathrm{T}}^{b}\right)=v_{n}\left(p_{\mathrm{T}}^{a}\right) v_{n}\left(p_{\mathrm{T}}^{b}\right) .
$$

Figure 2 first column shows that the shape and nature of the 1D azimuthal correlation vary with spherocity, and one can observe that the amplitude of the correlation is greater in low- $S_{0}$, intermediate in $S_{0}$-integrated and lower in high- $S_{0}$ events. Again, the amplitude of the correlation is larger in semi-central collisions compared to most central collisions. Figure 2 second column represents the two particle elliptic flow co-efficient $v_{2,2}\left(p_{T}^{a}, p_{T}^{b}\right)$ as a function of spherocity. Here, $v_{2,2}\left(p_{T}^{a}, p_{T}^{b}\right)$ has strong centrality dependence with showing greater values towards semi-central collisions. As far as spherocity is concerned, the high- $S_{0}$ events are found to have nearly zero elliptic flow while the low- $S_{0}$ events contribute significantly to elliptic flow of spherocity-integrated events. This is evident from the third column of Fig. 2.

\section{Summary}

In summary, we found that transverse spherocity successfully differentiates heavy-ion collisions' event topology based on their geometrical shapes, i.e. high and low values of spherocity $\left(S_{0}\right)$. The high- $S_{0}$ events are found to have nearly zero elliptic flow, while the low- $S_{0}$ events contribute significantly to the elliptic flow of spherocity-integrated events. Transverse spherocity is anti-correlated to elliptic flow.

\section{Acknowledgements}

R.S. acknowledges the financial supports under the CERN Scientific Associateship and the financial grants from DAE-BRNS Project No. 58/14/29/2019-BRNS. The authors would like to acknowledge the usage of resources of the LHC grid computing facility at VECC, Kolkata, and the computing farm at ICN-UNAM. S.T. acknowledges the support from INFN postdoctoral fellowship in experimental physics. A.O. acknowledges the financial support from CONACyT under Grant No. A1-S-22917.

\section{References}

[1] V. Khachatryan et al. [CMS], Phys. Lett. B 765 (2017), 193

[2] J. Adam et al. [ALICE], Nature Phys. 13 (2017), 535 
[3] E. Cuautle, R. Jimenez, I. Maldonado, A. Ortiz, G. Paic and E. Perez, [arXiv:1404.2372 [hep-ph]].

[4] A. Ortiz, G. Paić and E. Cuautle, Nucl. Phys. A 941 (2015), 78

[5] G. P. Salam, Eur. Phys. J. C 67 (2010), 637

[6] G. Bencédi [ALICE], Nucl. Phys. A 982 (2019), 507

[7] S. Acharya et al. [ALICE], Eur. Phys. J. C 79 (2019), 857

[8] G. Aad et al. [ATLAS], Phys. Rev. C 92 (2015), 034903

[9] M. Masera, G. Ortona, M. G. Poghosyan and F. Prino, Phys. Rev. C 79 (2009), 064909

[10] N. Mallick, R. Sahoo, S. Tripathy and A. Ortiz, J. Phys. G 48 (2021), 045104

[11] Z. W. Lin, C. M. Ko, B. A. Li, B. Zhang and S. Pal, Phys. Rev. C 72 (2005), 064901

[12] S. A. Voloshin, A. M. Poskanzer and R. Snellings, Landolt-Bornstein 23 (2010), 293

[13] G. Aad et al. [ATLAS], Phys. Rev. C 86 (2012), 014907

[14] S. Chatrchyan et al. [CMS], Phys. Rev. C 89 (2014), 044906

[15] K. Aamodt et al. [ALICE], Phys. Lett. B 708 (2012), 249 